\title{
LA ESTRUCTURA COGNITIVA DE LOS TRASTORNOS DE PERSONALIDAD DE EVITACIÓN Y DEPENDIENTE: UN ESTUDIO CON LA TÉCNICA DE REJILLA
}

\author{
Guillem FeiXas, Mónica ANTEQuera, Gala Almuedo y LoRenzo Bach \\ Facultad de Psicología, Universidad de Barcelona
}

\begin{abstract}
Resumen: En este artículo se estudia la estructura cognitiva de una muestra de participantes diagnosticados de trastornos de personalidad por dependencia $(n=24)$ y por evitación $(n=21)$ de forma conjunta mediante una serie de índices derivados de la Técnica de Rejilla. Esta técnica está fundamentada en la Teoría de Constructos Personales y estudia de forma sistemática la manera en la que la persona construye los significados acerca de sí mismo y los demás. Al comparar estos participantes con una muestra de voluntarios sin diagnóstico $(n=45)$, apareada en cuanto a edad y sexo, se encuentran diferencias significativas en cuanto a su menor complejidad cognitiva (diferenciación), y una tendencia a presentar más relaciones inconsistentes entre los constructos obtenidos en la rejilla. En cuanto a la construcción de sí mismos, hallamos menor autoestima, un mayor aislamiento social autopercibido, e incluso una cierta tendencia a mostrar un patrón de resentimiento.
\end{abstract}

Palabras clave: Trastorno de personalidad por dependencia, trastorno de personalidad por evitación, teoría de los constructos personales, técnica de rejilla, complejidad cognitiva, diferenciación, autoestima, conflictos cognitivos.

\author{
Cognitive structure of avoidant and dependent \\ personality disorders: A study with the Repertory Grid
}

\begin{abstract}
We study the cognitive structure of a conjoint sample of patients with a diagnosis of dependent $(n=24)$ and avoidant $(n=21)$ personality disorders using some measures derived from the Repertory Grid. This technique, based on Personal Construct Theory, aims at a systematic exploration of the ways in which people give meaning to their experience in their own terms. We compare the above clinical sample with a non-clinical sample (volunteers; $n=45$ ), paired by age and sex, and find significant differences suggesting that participants of the former show a lower level of cognitive complexity (differentiation) and a higher tendency towards conflict in terms of contradictory correlations among the constructs provided by them. With respect to self-construction, we find lower self-esteem, higher perceived social isolation, and a tendency to show a pattern of resentment.
\end{abstract}

Keywords: Dependent personality disorder, avoidant personality disorder, personal construct theory, repertory grid, cognitive complexity, differentiation, self-esteem, cognitive conflicts.

\section{INTRODUCCIÓN}

El DSM-IV-TR (APA, 2000) define los trastornos de personalidad como patrones permanentes e inflexibles de experiencia interna y de com-

Recibido 5 noviembre 2004; aceptado 5 septiembre 2006.

Correspondencia: Guillén Feixas, Departamento de Personalidad, Evaluación y Tratamiento Psicológicos, Facultad de Psicología, Universidad de Barcelona, Passeig Vall d'Hebron 171,08035 Barcelona. Correo-e: gfeixas@ub.edu portamiento que se apartan acusadamente de las expectativas de la cultura del sujeto, con los consecuentes perjuicios y malestar, y con un inicio relativamente temprano y desarrollo estable a través del tiempo. Esta bien establecido, tanto desde la investigación como desde la práctica clínica, que su presencia agrava la prognosis de los trastornos del eje I, resultando en un tratamiento más largo y costoso.

Este estudio se centra en los trastornos de personalidad por dependencia y por evitación, que 
comparten algunas características sustanciales. En concreto, el DSM-IV-TR los engloba en el grupo $\mathrm{C}$, junto con el trastorno obsesivo-compulsivo de la personalidad. Los participantes diagnosticados de trastorno por dependencia muestran dificultades para tomar decisiones cotidianas en ausencia de otro que los dirija o reafirme. De esta forma, tienden a ser pasivos y dejar que otra persona asuma la responsabilidad en áreas importantes de su vida. Por tanto, su autoestima es baja y se muestran llenos de sentimientos de insuficiencia.

Desde el punto de vista del déficit de las funciones metacognitivas (Semerari, 1999; Dimaggio y Semerari, 2004), los participantes con trastornos de personalidad por dependencia muestran dificultad, en ausencia de una persona de referencia, para representarse los propios fines, deseos, y planes para conseguirlos. Por otro lado, lejos de lograr un buen descentramiento, suelen mantener una perspectiva egocéntrica con la que atribuyen procesos mentales al otro generalmente del tipo; "yo soy el centro de lo que el otro piensa». Finalmente, sus estrategias de afrontamiento son poco elaboradas, limitándose a afrontar los problemas buscando el apoyo constante del otro.

Las personas con trastorno de personalidad por evitación temen la evaluación negativa de los demás, son hipersensibles aún a la menor crítica, no soportan hacer el ridículo y necesitan garantías de apoyo incondicional para mantener relaciones con los otros. Debido a sus escasas habilidades sociales y al temor a la evaluación negativa, recurren a estrategias de evitación. Estas personas tienen una autoestima muy baja, por lo que son frecuentes los episodios depresivos o los estados de ansiedad comórbidos. Sus déficit metacognitivos principales tienen que ver con dificultades para identificar los componentes de los estados mentales propios (emociones, deseos y metas) y relacionarlos con los de otras personas, lo que limita también su capacidad para el descentramiento (Dimaggio y Semerari, 2004).

Ciertamente, la inclusión de estos dos trastornos en el mismo grupo viene justificada por varios ingredientes comunes: baja autoestima, relaciones personales restrictivas y a menudo problemáticas, $y$ déficits metacognitivos en las áreas de autorreflexividad, diferenciación, descentramiento, y estrategias de afrontamiento primarias o directas.
Se han estudiado muchas de las características de estos trastornos, pero pocos son los estudios que se centran en la estructura y mecanismos cognitivos. Sin embargo, el papel de estos factores puede resultar clave, sobre todo si tenemos en cuenta que para Dimaggio y Semerari (2004) la personalidad es en gran medida la resultante de una serie de operaciones cognitivas:

«construir una imagen de sí mismo, del significado del mundo, relacionarse con los demás, y encontrar soluciones a los problemas que plantea el ambiente. Los mecanismos dedicados a estas operaciones pueden funcionar mal. Y si la disfunción se extiende a amplias áreas de la vida social $e$ interior asume la forma de un trastorno de personalidad.» (p. 5)

Para estudiar algunos estos aspectos cognitivos, la Técnica de Rejilla (TR) puede ser un método muy adecuado. Este instrumento fue ideado por George Kelly (1955), e inicialmente le llamó «Rep Test» en el contexto de su Teoría de los Constructos Personales (TCP). Kelly concibió al ser humano como un científico que construye hipótesis sobre sí mismo, los demás $y$ en definitiva, sobre el mundo que lo rodea. En el devenir de la experiencia, donde ocurren las emociones, los pensamientos y los comportamientos en un flujo continuo e interdependiente, es donde se ponen a prueba tales hipótesis, resultando en su validación o bien en su invalidación, y consecuente reconstrucción de significados (Botella y Feixas, 1998; Kelly, 2001).

La TCP se basa en una epistemología constructivista que propone que no existe una sola realidad verdadera y única sino que cada persona construye la suya propia, sus propios significados con los que organiza su entorno físico y social. Por supuesto, eso no equivale a afirmar que «todo vale», sino que la validez de una construcción está ligada a su capacidad y validez predictiva puesto que no puede establecerse de forma inequívoca su valor de «verdad». Además de la TCP, otros modelos cognitivos se han inspirado en la epistemología constructivista (véase las revisiones de Feixas y Villegas, 2000; Neimeyer y Mahoney, 1995) y han resultado influyentes en el campo de las terapias cognitivas (Feixas y Miró, 1993).

La TR es un instrumento de medida semiestructurado fundamentado en la obtención de los 
distintos constructos de la persona a estudiar (Feixas y Cornejo, 1996; Fransella, Bell y Bannister, 2004; Kelly, 1955; Rivas y Marco, 1985). Es decir, no es un test estándar en el que cada sujeto tiene que contestar las mismas preguntas sino que cada uno elabora de alguna manera los «ítems» que se emplearán. Se trata, por tanto, de una técnica idiosincrásica, centrada en la persona; al contrario de la gran mayoría de instrumentos que están centrados en la perspectiva del investigador. También se considera la TR como una de las principales técnicas subjetivas (Feixas, 2003) o de evaluación constructivista (Neimeyer, 1993).

Teniendo en cuenta que ambos trastornos de personalidad objetos de nuestro estudio (por dependencia y por evitación) presentan características similares se ha decidido compararlos con un grupo de voluntarios sin diagnóstico para observar si existen diferencias significativas a partir del análisis de su estructura cognitiva mediante la Técnica de Rejilla.

\section{MÉTODO}

\section{Participantes}

Este estudio supone un reanálisis de los datos del trabajo de Feixas, Bach y Laso (2004). La muestra utilizada se recogió desde 1989 hasta 1999 , y consta de 726 participantes, de los cuales 422 completaron la Técnica de Rejilla (TR) en un contexto clínico (centros de psicoterapia privados del área de Barcelona). Para nuestros propósitos se han seleccionado 90 participantes en función de los siguientes criterios.

Un primer grupo, denominado grupo clínico $(n=45)$, consta de dos tipos de participantes: los que cumplen los criterios del DSM-IV en trastorno de la personalidad por dependencia $(n=24)$, y los que cumplen los de trastorno de la personalidad de evitación ${ }^{1}(n=21)$. En un estudio preliminar, se compararon los dos tipos de pacientes con trastornos de personalidad (por dependencia y por evitación) del grupo clínico y no se hallaron diferencias significativas en las variables a estudiar entre las dos tipologías de participantes. Este es el motivo por el que se han tratado conjuntamente.

\footnotetext{
${ }^{1}$ Ambos grupos de criterios no han sufrido modificaciones en la versión más reciente del DSM-IV-TR.
}

Un segundo grupo de participantes, denominado grupo control $(n=45)$, procede de la población normal, pudiéndose distinguir, dentro de dicho grupo, dos tipos de participantes. Por un lado voluntarios reclutados por estudiantes de psicología (tanto licenciados como no licenciados) en sus ambientes naturales (como parte de un trabajo práctico y posterior al entrenamiento en la administración de la TR) $(n=34)$ y, por otro lado, participantes que pidieron ayuda psicológica en distintos centros privados de Barcelona, pero que no cumplían los criterios para diagnosticar ningún trastorno $(n=11)$. Sus motivos de consulta se correspondian con lo que en el DSM-IV-TR se engloba bajo el epígrafe de «otros problemas que pueden ser objeto de atención clínica». La elección de los participantes del grupo control viene dada por su emparejamiento con los participantes del grupo clínico en las variables: edad, y sexo. Como en muchos casos se contaba con varios participantes de la misma edad y sexo que los del grupo clínico, se seleccionaron de entre éstos al azar.

En el grupo clínico hay una media de edad de 31,69 años $(D T=8,09)$, con un rango de edad de los 20 a los 54 años, y en el grupo control hay una media de edad de 31,67 años $(D T=8,19)$, con un rango de edad de los 20 a los 55 años. En cuanto al sexo, en ambos grupos hay $62 \%$ de mujeres y $38 \%$ de hombres.

\section{Instrumento y medidas}

Para ambos grupos se utilizó la Técnica de Rejilla, un instrumento de evaluación de las dimensiones y estructura del significado personal, ideado por George Kelly (1955) dentro de su Teoría de Constructos Personales (TCP). Lo más singular de la TR es que pretende estudiar a la persona, o como ésta da un sentido a su experiencia, en sus propios términos (véase Feixas y Cornejo; 1996, Feixas y Villegas, 2000, para más información). No se trata de un test centrado en los constructos teóricos del investigador sino que se sitúa en una perspectiva centrada en la persona, en el estudio de sus teorías implícitas (sus constructos personales, según Kelly). 
Existen varias modalidades de diseño de rejilla, la que se utilizó fue la TRI (Técnica de Rejilla Interpersonal). Ésta tiene formato de cuadrícula, donde en las columnas se colocan los elementos, en las filas los constructos y las puntuaciones en las casillas que forman cada cruce de constructo por elemento. Como elementos se introducen entre 10 y 20 personas significativas del mundo interpersonal del sujeto, incluyendo un elemento «yo actual», uno "yo ideal» (cómo me gustaría ser) y otro «persona non grata» (persona que no me gusta cómo es).

Los constructos se elaboran en forma bipolar, es decir, una característica que la persona atribuye a un elemento diferenciándola con respecto a su polo opuesto. Se obtienen a través de sucesivas preguntas acerca de las similitudes y diferencias entre pares de elementos (véase Feixas y Cornejo, 1996; Feixas, De la Fuente y Soldevila, 2003 para más información). Por ejemplo, se pregunta acerca de las similitudes entre el elemento «madre» y el elemento "padre», el sujeto puede contestar que los dos son «luchadores» (como opuesto a "vagos»), y así sucesivamente hasta que no surjan más constructos. El número total de constructos, por tanto, puede variar de una persona a otra ya que se promueve una elicitación de los constructos sin más limitaciones que las inevitables derivadas de la propia situación de entrevista.

Una vez obtenidos los constructos se aplican, uno a uno, a cada elemento en función de una escala tipo Likert de 7 puntos, de forma bipolar ${ }^{2}$. El resultado es una matriz numérica de resultados que puede ser analizada cualitativamente, $o$ bien cuantitativamente mediante el programa informático RECORD v. 2.0 (Feixas y Cornejo; $1996)^{3}$, obteniendo los siguientes índices de medida cognitivos:

a. Índices de medida de la estructura cognitiva: (1) Porcentaje de Varianza Explicado por el Primer Factor (PVEPF). Indica la magnitud de la principal dimensión de significado. Su cálculo está basado en el análisis factorial de la matriz

\footnotetext{
${ }^{2}$ Si se aplica el polo izquierdo del constructo (p.ej., «luchadon) se pueden emplear las puntuaciones « $1 »$ («muy luchador»), «2» («bastante luchador»), y «3» («un poco luchadon)). Si el polo adecuado según el sujeto es el opuesto, las puntuaciones serían «5» (un poco vago»), «6» (bastante
}

de datos de la rejilla y se considera el mejor indicador de complejidad cognitiva (diferenciación). Si esta dimensión tiene un valor alto señala que la matriz de datos puede reducirse a una sola dimensión y aún asi explicar mucha varianza. Esto puede resultar un indicador de un cierto grado de unidimensionalidad del sujeto al construir su mundo interpersonal. En cambio, si el valor es menor señala que hay una buena proporción de varianza disponible para otras dimensiones que desempeñan un papel en los procesos de construcción de la persona. (2) Número de constructos. Se considera también una medida de complejidad cognitiva (diferenciación) aunque, probablemente, también asociada a factores de capacidad verbal (Feixas, Bach y Laso, 2004). (3) Conflictos cognitivos. Aunque hemos estudiado otros tipos de conflictos cognitivos (Feixas y Saúl, 2004), en este trabajo nos centramos en los de naturaleza triádica. Este tipo de conflictos, propuesto por Slade y Sheehan (1979), se computan según el número de tríadas de constructos desequilibradas, es decir, cuando se dan correlaciones negativas entre tres constructos, o bien otros patrones de correlaciones que se contradicen entre sí. A diferencia del cómputo original, ya en otros trabajos (Feixas, Bach y Laso, 2004) empleamos como punto de corte una correlación de 0,20 para no contabilizar los conflictos triviales que detectan correlaciones muy próximas a cero. Un ejemplo de conflicto entre tres constructos personales se puede observar en la Figura 1.

b. Indices de medida de la construcción del si mismo: (1) Diferenciación entre el "yo actual» y el «yo ideal». Se calcula mediante la correlación entre estos dos elementos y puede considerarse una medida de la autoestima. (2) Diferenciación entre el «yo actual» $y$ «otros». Se calcula a partir de la correlación entre el elemento «yo actual» y un elemento artificial llamado «otros», resultado de promediar las puntuaciones de todos los elementos menos el «yo actual» y el «yo ideal». Se puede considerar que las puntuaciones muy bajas o negativas son indicadoras de «aislamiento social autopercibido", en cambio las puntua-

vago»), y «7» («muy vago»). En el caso de no poder decidirse por ninguno de los dos polos se puntúa un «4».

${ }^{3}$ En la actualidad ya existe la versión 4.0 del programa RECORD disponible en Internet: www.terapiacognitiva.net/record 
vive en el aire

(vs. se da cuenta de los problemas)

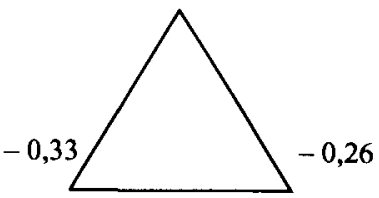

equilibrada $\quad-0,40 \quad$ pesimista

(vs. tiene prontos) (vs. optimista)

Figura 1. Representación gráfica de un conflicto (Feixas y Cornejo, 1996). Los coeficientes indicados son correlaciones de Pearson.

ciones positivas de «identificación». (3) Diferenciación entre el «yo ideal» $y$ «otros». Se calcula mediante la correlación entre estos dos elementos, $y$ puede considerarse una medida de adecuación percibida en los otros. Mientras que una puntuación positiva indica que se tiene una visión de que «todos son buenos», una puntuación negativa o muy baja muestra que esta persona está insatisfecha con la gente que le rodea. En estas tres variables que hacen referencia a correlaciones se ha efectuado primero la transformación $\mathrm{z}$ de Fisher ${ }^{4}$ para poder incluirlas en los análisis estadísticos.

\section{Procedimiento}

Los datos de las rejillas del estudio de Feixas, Bach y Laso (2004) fueron analizados mediante el programa RECORD v. 2.0 (Feixas y Cornejo, 1996) y los resultados de cada par- ticipante en las variables antes mencionadas se incluyeron en una base de datos y se transformaron posteriormente en un fichero de datos para ser tratado estadísticamente mediante el programa SPSS 11.5. Para el presente estudio se han creado las categorizaciones pertinentes en dicho fichero y se ha realizado la prueba de comparación de medias para muestras independientes ( $T$ de Student) utilizando el mismo programa. Posteriormente, se ha ejecutado una regresión logística binaria con la intención de conocer qué variables son las más importantes a la hora de predecir la pertenencia al grupo clínico vs. el grupo control.

\section{RESULTADOS}

En la Tabla 1 se presentan las medias y desviaciones típicas de distintos índices de la TR,

Tabla 1. Medias, desviaciones típicas y significación estadística de los índices de medida cognitivos.

\begin{tabular}{|c|c|c|c|}
\hline & \multicolumn{2}{|c|}{ Media $(D T)$} & \multirow[b]{2}{*}{$t(88)$} \\
\hline & Grupo control & Grupo clínico & \\
\hline PVEPF & $41,56(10,50)$ & $48,01(9,76)$ & $-3,02^{* *}$ \\
\hline Diferenciación «yo actual - yo ideal» & $0,41(0,55)$ & $-0,12(0,46)$ & $4,92 * * *$ \\
\hline Diferenciación «yo actual - otros» & $0,22(0,40)$ & $-0,13(0,38)$ & $2,87^{* *}$ \\
\hline Diferenciación «yo ideal - otros» & $0,33(0,39)$ & $0,18(0,39)$ & 1,87 \\
\hline Conflictos cognitivos & $0,01(0,01)$ & $0,01(0,01)$ & $-1,68$ \\
\hline Número de constructos & $15,89(3,80)$ & $13,44(2,50)$ & $3,60^{* *}$ \\
\hline
\end{tabular}

Nota. PVEPF $=$ Porcentaje de varianza explicada por el primer factor. ${ }^{* *} p<0,01,{ }^{* * *} p<0,001$,

${ }^{4}$ La transformación de Fisher se utilizó para poder tratar datos de correlaciones ya que éstas no se distribuyen normalmente. Una vez realizada la transformación ya se pueden llevar a cabo los análisis estadísticos correspondientes. 
comparando el grupo clínico (compuesto por personas con diagnóstico de Trastorno de Personalidad por Dependencia y por Evitación) con el grupo control. Como se puede observar existen diferencias significativas entre ambos grupos en todos los indices utilizados excepto en la diferenciación entre el «yo ideal» y «otros», y en el índice de conflictos cognitivos. Sin embargo, en ambos casos, se aprecia una cierta tendencia que se aproxima al nivel de significación.

A continuación realizamos una regresión logística binaria tomando como variable criterio la pertenencia a la muestra de trastorno de personalidad (por evitación o por dependencia, codificada como 1) o la pertenencia a una muestra de población normal (codificada como 0 ). Las variables independientes tenidas en cuenta son el PVEPF, el número de constructos, la diferenciación yo-ideal, la diferenciación yo-otros y la diferenciación idealotros. El método de selección de variables ha sido el método pasos hacia delante (Wald) y se ha completado en 3 pasos. El porcentaje de casos correctamente clasificados ha sido del $77,8 \%$, con una $R^{2}$ de Nagelkerke de 0,429 $\left(\chi^{2}=40,15, g . l .=5, p<0,001\right)$, lo que indica que la proporción de variación en la variable

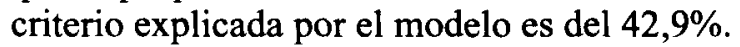

Tal como muestra la Tabla 2, las variables que son significativas en el modelo son, por este orden, la diferenciación yo-ideal, el número de constructos y el PVEPF. Por tanto, la mayor probabilidad de pertenecer al grupo con trastorno de personalidad (por evitación o por dependencia) se da cuando los participantes presentan una baja autoestima (baja corre- lación yo-ideal) y una menor diferenciación cognitiva (menor número de constructos y un PVEPF más elevado).

\section{DISCUSIÓN Y CONCLUSIONES}

Por lo que respecta a los índices estructurales, parece claro que los participantes diagnosticados por trastorno de personalidad por dependencia y por evitación presentan una menor complejidad cognitiva (entendida como diferenciación) que la población general. Se constata que en el proceso de administración aportan menos constructos y que éstos están más intensamente asociados entre sí. Ello sugiere una estructura más simple, o incluso se podría decir más rígida, y por tanto, más susceptible de ser invalidada ante la complejidad que puede aportar la experiencia. Esta falta de complejidad de la estructura cognitiva podría estar relacionada con los déficit metacognitivos de diferenciación señalados por el grupo de Semerari (1999; Dimaggio y Semerari, 2004).

La posibilidad de que los pacientes diagnosticados por estos trastornos de personalidad dispongan de menor complejidad cognitiva coincide también con las observaciones clínicas de Soldz (1986), quien sugiere que estos pacientes operan sobre sistemas de construcción relativamente globales, indiferenciados y desintegrados, particularmente en el dominio de las relaciones interpersonales. Por esta razón, un terapeuta bien intencionado que pretenda invalidar aspectos centrales de su sistema de creencias patológico puede frustrarse, porque, a diferencia de otros más sanos, estos pacientes no tienen sistemas inter-

Tabla 2. Detalle de datos estadísticos de los diferentes pasos efectuados mediante el análisis de regresión logística binaria

\begin{tabular}{llccc}
\hline & & $B$ & Wald (1) & Odds ratio \\
\hline Paso 1 & Dif. «yo actual - yo ideal» & $-2,30$ & $15,4^{* * *}$ & 0,100 \\
Paso 2 & Número de constructos & $-0,21$ & $6,7^{* *}$ & 0,805 \\
& Diferenciación «yo actual - yo ideal» & $-2,27$ & $13,2^{* * *}$ & 0,103 \\
Paso 3 & PVEPF & 0,05 & $4,1^{*}$ & 1,060 \\
& Número de constructos & $-0,18$ & $4,6^{*}$ & 0,832 \\
& Diferenciación «yo actual - yo ideal» & $-2,22$ & $11,6^{* *}$ & 0,109 \\
\hline
\end{tabular}

Nota: $\mathrm{PVEPF}=$ Porcentaje de varianza explicada por el primer factor. ${ }^{*} p<0,05,{ }^{* *} p<0,01,{ }^{* * *} p<0,001$ 
pretativos alternativos para ser utilizados. Además, casi todas las predicciones sociales involucran sus constructos nucleares de una manera relativamente directa. Así, la anticipación de la desconfirmación de sus sistemas de interpretación poco complejos y diferenciados, rígidos aunque frágiles, puede precipitarlos de repente a la ansiedad de un mundo social imposible de interpretar, y la amenaza del cambio en sus estructuras más fundamentales de identidad (Botella y Feixas, 1998; Kelly, 1955).

En cuanto a la coherencia interna de los constructos aportados en la administración de la TR, los participantes del grupo clínico presentan una tendencia a tener más correlaciones conflictivas entre constructos. Ello quiere decir que un mismo constructo puede estar asociado a la vez a aspectos positivos y negativos en función de otros constructos. Por tanto, se puede suponer que estos conflictos pueden propiciar algunos bloqueos en la toma de decisiones o sentimientos ambivalentes.

En comparación con el grupo control, y como era de prever, la construcción del sí mismo de estos participantes diagnosticados por trastorno de personalidad está marcada por una baja autoestima. También por un aislamiento social autopercibido (aunque la diferenciación "yo actual-otros» no aparece en el modelo de la regresión logística). Esto hace suponer la existencia de sentimientos de inadecuación personal a la vez que la sensación de ser distinto a los demás. Por otro lado, se observa una tendencia a percibir a los demás también como inadecuados. Feixas y Cornejo (1996) sugieren que esta combinación dada por baja autoestima, elevado aislamiento social autopercibido y baja adecuación de los demás, configura un perfil de resentimiento. Como comentan Feixas, De la Fuente y Soldevila (2003), «es frecuente que este tipo de participantes relacionen su malestar con las actitudes de los otros hacia ellos y experimenten sentimientos de rencor y rabia»" (p. 166). Aunque este hallazgo se había observado ya en la clínica,

El presente estudio sobre el análisis de los trastornos de la personalidad mediante la TR es el primero que existe de tales características, a pesar de que actualmente se encuentran numerosos trabajos sobre trastornos de perso- nalidad y también otros que emplean la TR. Se ha podido comprobar que la combinación de estos estudios puede aportar datos importantes tanto para aquéllos interesados en los trastornos de personalidad como para los que estudian la TR y su aplicabilidad. Por tanto, se trata de un estudio pionero a partir del cual pueden derivarse nuevas investigaciones que ayuden a incrementar los conocimientos sobre estos trastornos y mejorar los tratamientos psicológicos a partir de centrarse en la construcción del sí mismo y en la estructura cognitiva de los pacientes.

\section{REFERENCIAS}

American Psychology Association (APA) (2000). Diagnostic and statistical manual of mental disorders: DSM-IV-TR. Washington, DC: APA.

Botella, L., y Feixas, G. (1998). Teoría de los constructos personales: Aplicaciones a la práctica psicológica. Barcelona: Laertes.

Dimaggio, G. y Semerari, A. (2004). I disturbi di personalità: Modelli e trattumento. Roma: Laterza.

Feixas, G. (2003). Subjective methods. En R. Fernandez-Ballesteros (ed.), The Enciclopedia of Psychological Assessment (Vol. II, págs. 937-943). London: Sage.

Feixas, G., Bach, L., y Laso, E. (2004). Factors affecting interpersonal construct differentiation when measured using the Repertory Grid. Journal of Constructivist Psychology, 17, 297-311.

Feixas, G. y Cornejo, J. M. (1996). Manual de la técnica de rejilla mediante el programa RECORD ver. 2.0 ( $2^{\mathrm{a}}$ ed.). Barcelona: Paidós.

Feixas, G., De la Fuente, M. y Soldevila, J. M. (2004). La técnica de rejilla como instrumento de evaluación y formulación de hipótesis clínicas. Revista de Psicopatología y Psicología Clinica, 8 , 153-172.

Feixas, G. y Miró, M.T. (1993). Aproximaciones a la psicoterapia: una introducción a los tratamientos psicológicos. Barcelona: Paidós.

Feixas, G. y Saúl, L. A. (2004). The Multi-Center Dilemma Project: An investigation on the role of cognitive conflicts in health. Spanish Journal of Psychology, 7, 69-78.

Feixas, G., y Villegas, M. (2000). Constructivismo y Psicoterapia ( $3^{\mathrm{a}}$ ed.). Bilbao: Desclée de Brouwer.

Fransella, F., Bell, R., y Bannister, D. (2004). A manual of repertory grid technique $\left(2^{\mathrm{a}} \mathrm{ed}\right.$.). Chichester, Reino Unido: Wiley. 
Kelly, G. A. (1955). The Psychology of personal constructs (2 vols.). New York: Norton.

Kelly, G. A. (2001). Psicología de los constructos personales: Textos escogidos (B. Maher, compilación en inglés; G. Feixas, editor). Barcelona: Paidós.

Neimeyer, G. (comp.) (1993). Evaluación constructivista. Barcelona: Paidós, 1996.

Neimeyer, R. y Mahoney. M. (Eds.) (1995). Constructivismo en psicoterapia. Barcelona: Paidós, 1998.

Rivas, F., y Marco, R. (1985). Evaluación conductual subjetiva: La técnica de rejilla. Valencia: Centro Editorial de Servicios y Publicaciones Universitarias.
Semerari, A. (1999). Psicoterapia cognitiva del paziente grave. Metacognizione e relazione terapeutica. Milano: Raffaello Cortina Editore (trad. castellana en Desclée de Brouwer, Bilbao, 2002).

Slade, P. D., y Sheehan, M. J. (1979). The measurement of "conflict» in repertory grids. British Journal of Psychology, 7, 519-524.

Soldz, S. (1986). Construing of others in psychotherapy: Personal construct perspectives. Journal of Contemporary Psychotherapy, 16, 52-61. 